\title{
ARTINIAN SUBRINGS OF A COMMUTATIVE RING
}

\author{
ROBERT GILMER AND WILLIAM HEINZER
}

\begin{abstract}
Given a commutative ring $R$, we investigate the structure of the set of Artinian subrings of $R$. We also consider the family of zero-dimensional subrings of $R$. Necessary and sufficient conditions are given in order that every zero-dimensional subring of a ring be Artinian. We also consider closure properties of the set of Artinian subrings of a ring with respect to intersection or finite intersection, and the condition that the set of Artinian subrings of a ring forms a directed family.
\end{abstract}

\section{INTRODUCTION}

Suppose $R$ is a commutative ring with unity element. We consider here various properties of the family $\mathscr{A}$ of Artinian subrings of $R$ and the family $\mathscr{Z}$ of zero-dimensional subrings of $R$. We remark that the inclusion $\mathscr{A} \subseteq \mathscr{Z}$ may be proper, even if $R$ is Noetherian; for example, Corollary 3.4 implies that if $K$ is an infinite field of positive characteristic, then the local principal ideal ring $K[X] /\left(X^{2}\right)$ contains a zero-dimensional subring that is not Artinian. Of course, if every subring of $R$ were Noetherian, the families $\mathscr{A}$ and $\mathscr{Z}$ would be identical. Thus one source of motivation for this work comes from papers such as $\left[\mathrm{G}_{1}, \mathrm{~W}_{1}, \mathrm{~W}_{2}, \mathrm{GH}_{1}, \mathrm{GH}_{3}\right]$ that deal with Noetherian and zerodimensional pairs of rings, hereditarily Noetherian rings, and hereditarily zerodimensional rings. Another source of motivation is related to our work in $\left[\mathrm{GH}_{3}\right]$, where we considered several problems concerning a direct product of zero-dimensional rings. One result (Theorem 6.7) in $\left[\mathrm{GH}_{3}\right]$ poses the problem of determining equivalent conditions on a zero-dimensional quasilocal ring $S$ in order that the family of all Artinian subrings of $S$ should form a directed family; we address this problem in $\S 5$.

Section 2 deals with the problem of determining what rings contain an Artinian or a zero-dimensional subring. In $\S 3$ we determine in Theorem 3.2 those pairs $R \subseteq S$ of rings with $R$ Artinian such that each zero-dimensional subring

Received by the editors July 9, 1990 and, in revised form, December 9, 1990.

1980 Mathematics Subject Classification (1985 Revision). Primary 13E10; Secondary 13A15, 13A99, 13B02, 13E05, 16A30.

Key words and phrases. Artinian subring, zero-dimensional subring, directed union of Artinian subrings, Noetherian ring, finite spectrum, hereditarily Noetherian ring, zero-dimensional pair, coefficient field, coefficient ring, absolutely algebraic field. Study.

Work of the first author was partially done while the author visited the Institute for Advanced

Research of the second author was partially supported by NSF Grant DMS 8800762 . 
of $S$ containing $R$ is Artinian, and Theorem 3.6 characterizes those rings $T$ such that each zero-dimensional subring of $T$ is Artinian.

Section 4 treats the question of whether the set $\mathscr{A}$ of Artinian subrings of $R$ is closed under finite or arbitrary intersection. For any ring $R$, the set $\mathscr{Z}$ of zero-dimensional subrings of $R$ is closed under arbitrary intersection $\left[\mathrm{GH}_{4}\right]$, and it is known that $\mathscr{A}$ is closed under arbitrary intersection if $R$ is reduced. We show that in general $\mathscr{A}$ is not closed under finite intersection. Moreover, by using properties of Steinitz numbers, absolutely algebraic fields, and coefficient fields, we prove in Theorem 4.6 that $\mathscr{A}$ may be closed under finite intersection, but not under arbitrary intersection.

Under what conditions is the set $\mathscr{A}$ directed? This question is addressed in $\S 5$, which contains Corollary 5.11, the main result of the paper. Corollary 5.11 states that if $T$ is a hereditarily zero-dimensional ring with $\operatorname{Spec} T$ finite, then $\mathscr{A}$ is directed, and in fact, $\mathscr{A}$ is closed under finite compositum. The proof of Corollary 5.11 uses results of Cohen $\left[\mathrm{C}_{1}\right]$ concerning the structure of complete local rings.

All rings we consider are commutative and have a unity element. If $R$ is a subring of $S$, we assume the unity of $S$ is contained in $R$, and hence is the unity of $R$. We use char $R$ to denote the characteristic of a ring $R$, and $|X|$ to denote the cardinality of a set $X$. If $R$ is a ring, we use $N(R)$ to denote the nilradical and $J(R)$ the Jacobson radical of $R$. The ring $R$ is said to be reduced if $N(R)=(0)$. If $R$ is a subring of a ring $S$, then an $S$-overring of $R$ is a subring of $S$ containing $R$.

We use $\mathbb{Z}$ and $\mathbb{Z}^{+}$, respectively, to denote the set of integers and the set of positive integers, and $\mathbb{Q}$ to denote the field of rational numbers.

If $R$ is a subring of a ring $S$, then $(R, S)$ is said to be a zero-dimensional pair if each subring of $S$ containing $R$ is zero-dimensional. If $R$ is a zerodimensional subring of a zero-dimensional ring $S$, it is observed in $\left[\mathrm{CH}_{3}\right]$ that (i) $(R, S)$ is a zero-dimensional pair if $S$ is a finitely generated $R$-algebra, and (ii) $(R, S)$ is a zero-dimensional pair if and only if $S$ is integral over $R$. If $\pi$ is the prime subring of a ring $R$, and if $(\pi, R)$ is a zero-dimensional pair, then the ring $R$ is said to be hereditarily zero-dimensional.

We acknowledge with thanks several helpful comments Mark Johnson has made regarding the contents of this paper.

\section{ZERO-DIMENSIONAL AND ARTINIAN SUBRINGS}

If a ring $R$ has positive characteristic, then the prime subring $\pi$ of $R$ is an Artinian ring, so the family of Artinian subrings of $R$ is nonempty. However, many rings of characteristic zero, such as $\mathbb{Z}$, do not admit any Artinian or even any zero-dimensional subrings. We begin (Proposition 2.2) by observing that this also holds for finitely generated extension rings of $\mathbb{Z}$, but first we record the following well-known and useful fact in the consideration of subrings and extension rings of a ring.

Result 2.1. If $R$ is a subring of a ring $S$ and $P$ is a minimal prime of $R$, then there exists a prime ideal $Q$ of $S$ such that $Q \cap R=P$. Thus there exists a minimal prime $Q$ of $S$ with this property. If $R$ is zero-dimensional, then the map $\operatorname{Spec}(S) \rightarrow \operatorname{Spec}(R)$ associated with the extension $R \subseteq S$ is surjective. 
Proof. This follows immediately from the observation that $U=R-P$ is a multiplicatively closed subset of $S$.

Proposition 2.2. If $R$ is a Hilbert ring of positive dimension and $T$ is a finitely generated extension ring of $R$, then $R$ admits no zero-dimensional $T$-overring. In particular, if $T$ is a finitely generated extension ring of $\mathbb{Z}$, then $T$ does not contain a zero-dimensional subring.

Proof. Assume that $S$ is a zero-dimensional $T$-overring of $R$. Let $P_{0}$ be a minimal prime of $R$ such that $\operatorname{dim}\left(R / P_{0}\right)>0$. By Result 2.1 , there exists a prime ideal $P$ of $S$ such that $P \cap R=P_{0}$, and a prime ideal $Q$ of $T$ such that $Q \cap S=P$. Since $S$ is zero-dimensional, if $M$ is a maximal ideal in $T$ that contains $Q$, then $M \cap S=P$. Therefore $M \cap R=P_{0}$, so that $T / M$ is a field and an extension ring of $R / P_{0}$. Since $R / P_{0}$ is Hilbert, it follows that $T / M$ is not finitely generated as an algebra over $R / P_{0}$ (cf. $\left[\mathrm{G}_{3}\right.$, Theorem 31.8]), and therefore $T$ is not finitely generated as an extension ring of $R$.

The statement concerning $\mathbb{Z}$ in Proposition 2.2. can be generalized as follows. If $T$ is a ring, then as in $\left[\mathrm{GH}_{3}\right]$, we denote by $\mathscr{C}(T)$ the set of numbers that are characteristics of residue fields of $T$. Observe that for a positive prime integer $p \in \mathbb{Z}, p \notin \mathscr{C}(T)$ if an only if $p$ is a unit of $T$; hence if $H$ is the multiplicative system in $\mathbb{Z}$ generated by $\{p: p \notin \mathscr{C}(T)\}$, then $\mathbb{Z}_{H}$ is a subring of $T$. If $\mathscr{C}(T)$ is infinite, then $\mathbb{Z}_{H}$ is a Hilbert ring of positive dimension, and hence by Proposition 2.2, $T$ has no zero-dimensional subring if $T$ is finitely generated over $\mathbb{Z}_{H}$.

Proposition 2.3. If each residue field of the ring $T$ has characteristic zero, then $T$ contains an isomorphic copy of $\mathbb{Q}$. In particular, a zero-dimensional quasilocal ring $T$ of characteristic zero contains an isomorphic copy of $\mathbb{Q}$.

Proof. In the notation of the paragraph preceding the statement of $(2.3), \mathscr{C}(T)$ $=\{0\}$ and $H=\mathbb{Z}-(0)$. Therefore $\mathbb{Z}_{H}=\mathbb{Q} \subseteq T$. If $T$ is zero-dimensional quasilocal of characteristic 0 , if $M$ is the maximal ideal of $T$, and if $\operatorname{char}(T / M)=c$, then the element $c \in T$ is nilpotent, hence 0 , and $\mathbb{Q}$ is contained in $T$ by the first assertion in (2.3).

Concerning the structure of zero-dimensional rings with finite spectrum, the following result is Corollary 5.5 of $\left[\mathrm{GH}_{3}\right]$.

Result 2.4. If $S$ is a zero-dimensional ring with finite spectrum, then there exists an Artinian subring $R$ of $S$ such that $(R, S)$ is a zero-dimensional pair. In particular, $S$ is a directed union of Artinian subrings.

Remark 2.5. (i) If a ring $R$ is indecomposable (or equivalently, if $\operatorname{Spec} R$ is connected), then any zero-dimensional subring of $R$ is quasilocal. This is immediate from the fact that an indecomposable ring has no nontrivial idempotents, while a zero-dimensional ring with more than one prime ideal has a nontrivial idempotent.

(ii) More generally, we note the following: (a) A ring with only a finite number of idempotents is a finite direct sum of indecomposable ideals (and conversely). (b) A finite direct product of $k$ nonzero indecomposable rings has exactly $2^{k}$ idempotents. It follows that if a ring $S$ is the direct sum of $n$ nonzero indecomposable ideals, then $S$ has exactly $2^{n}$ idempotent elements. If $R$ is a 
zero-dimensional subring of $S$, then $R$ has only finitely many idempotents, and hence, by (a), is a direct sum of $m$ nonzero indecomposable ideals, which must be quasilocal as rings by part (i) above. Hence $R$ has $m$ maximal ideals and $2^{m} \leq 2^{n}$ idempotents. Thus a zero-dimensional subring of $S$ has at most $n$ maximal ideals.

(iii) A zero-dimensional reduced ring $S$ with only finitely many idempotents is Artinian. This follows because (i) and (ii) imply that $S$ is a finite direct sum of indecomposable ideals $S_{i}$; each $S_{i}$ is zero-dimensional, quasilocal and reduced, and hence is a field.

An observation in $\left[\mathrm{GH}_{3}\right]$ is in the nature of a partial converse to the above remarks. It is true in general that if $R$ is a subring of $S$, then each nonzero element of $\mathscr{C}(S)$ is contained in $\mathscr{C}(R)$. Of course, it may happen that $0 \in$ $\mathscr{C}(S)-\mathscr{C}(R)$, (for example, consider $R=\mathbb{Z} \subseteq \mathbb{Q}=S$ ), and in general it may happen that $\mathscr{C}(S)$ is a proper subset of $\mathscr{C}(R)$, but $\mathscr{C}(R)=\mathscr{C}(S)$ if $R$ is zero-dimensional. Hence if $R$ is a zero-dimensional subring of $S$, then $R$ has at least $|\mathscr{C}(S)|$ maximal ideals. This implies that there exist zero-dimensional rings such as

$$
S=\prod\{\mathbb{Z} / p \mathbb{Z}: p \text { varies over an infinite number of distinct primes }\}
$$

that do not have any Artinian subrings. (If $S$ contained an Artinian subring $R$, then $\mathscr{C}(R)$ would be finite. But zero-dimensionality implies $\mathscr{C}(R)=\mathscr{C}(S)$, and $\mathscr{C}(S)$ is infinite.)

It is interesting to observe that another infinite product of finite rings yields a ring $T$ with $\mathscr{C}(T)$ finite such that $T$ does not contain a zero-dimensional subring.

Proposition 2.6. Let $p$ be a fixed prime number and let

$$
T=\prod_{n=1}^{\infty} \mathbb{Z} / p^{n} \mathbb{Z}
$$

Then $\mathscr{C}(T)=\{p\}$, but $T$ does not contain any zero-dimensional (and therefore any Artinian) subring.

Proof. Since $J(T)=\prod_{n=1}^{\infty} J\left(\mathbb{Z} / p^{n} \mathbb{Z}\right), p \in J(T)$, and hence $\mathscr{C}(T)=\{p\}$. Because $T$ has characteristic zero, the prime subring of $T$ is $\mathbb{Z}$. It is shown in $\left[\mathrm{GH}_{2}\right.$, Example 5] that if $I$ is the direct sum ideal

$$
I=\bigoplus_{n=1}^{\infty} \mathbb{Z} / p^{n} \mathbb{Z}
$$

and $S$ is the subring $\mathbb{Z}+I$ of $T$, then $S$ is an integral extension of $\mathbb{Z}$ that is not a subring of a zero-dimensional ring. If $T$ contains a zero-dimensional subring $R$, then the integral closure of $R$ in $T$ is still zero-dimensional and is a $T$-overring of $S$. This contradicts the fact that $S$ is not a subring of a zero-dimensional ring. Therefore $T$ does not contain any zero-dimensional subrings.

An alternative to the latter part of the proof above is the following. Observe that if $R$ is a zero-dimensional ring of characteristic zero, then $0 \in \mathscr{C}(R)$. This is clear from the fact that $\mathbb{Z} \subset R$ implies that there exists a prime of $R$ lying over $(0)$ in $\mathbb{Z}$. But as noted above, if $R$ is a zero-dimensional subring 
of a ring $T$, then $\mathscr{C}(R)=\mathscr{C}(T)$, which contradicts the fact also noted above that $\mathscr{C}(T)=\{p\}$.

Remark 2.7. If a ring $R$ admits a zero-dimensional (resp. Artinian) subring, then it is clear that any homomorphic image of $R$ also admits a zerodimensional (resp. Artinian) subring. From this observation and the fact that the families $\mathscr{A}$ and $\mathscr{Z}$ are closed under taking finite direct products, it follows that if $R=R_{1} \oplus \cdots \oplus R_{n}$, then $R$ has a zero-dimensional (resp. Artinian) subring if and only if each $R_{i}$ has a zero-dimensional (resp. Artinian) subring.

Concerning subrings of an indecomposable ring, the following is an immediate consequence of (2.3)-(2.5).

Result 2.8. Assume that $R$ is an indecomposable ring. If $R$ contains a zerodimensional subring, then $R$ also contains an Artinian subring. If $R$ is of characteristic zero and contains a zero-dimensional subring, then $\mathbb{Q}$ is a subring of $R$. If a ring $S$ is a finite direct sum of indecomposable ideals and if $S$ admits a zero-dimensional subring, then $S$ also admits an Artinian subring.

\section{RINGS FOR WHICH EACH ZERO-DIMENSIONAL SUBRING IS ARTINIAN}

If $T$ is a ring, then each Artinian subring of $T$ is, of course, zero-dimensional. The converse fails, but in this section we determine the class of rings $T$ for which, conversely, each zero-dimensional subring is Artinian. Following models in [ $\mathrm{W}_{1}, \mathrm{GH}_{1}, \mathrm{GH}_{3}$ ], we also consider this concept in the more general context of the set of all zero-dimensional $T$-overrings of a fixed subring $R$ of $T$. In this regard, we introduce two definitions. If $R$ is a subring of $T$, we say that the pair $(R, T)$ satisfies (ZA) if there exists a zero-dimensional $T$-overring of $R$ and if each zero-dimensional $T$-overing of $R$ is Artinian. We say that a ring $R$ satisfies (ZA) if the pair $(\pi, R)$ satisfies (ZA), where $\pi$ is the prime subring of $R$. We note that what might be called hereditarily Artinian rings and Artinian pairs of rings are characterized in Theorems 4.10 and 4.12, respectively, of $\left[\mathrm{GH}_{3}\right]$.

Result 3.1. If the pair $(R, T)$ satisfies (ZA), then $T$ has only finitely many idempotents.

Proof. Let $S$ be a zero-dimensional $T$-overring of $R$ and let $E$ be the set of idempotent elements of $T$. Then $S[E]$ is integral over $S$, hence zerodimensional, therefore Artinian. Consequently, $S[E]$ has only finitely many idempotents and the set $E$ is finite.

Theorem 3.2. Suppose $T$ is a ring and $R$ is an Artinian subring. Then $(R, T)$ satisfies (ZA) if and only if $T$ has only finitely many idempotents and the nilradical $N(T)=N$ of $T$ is a finitely generated $R$-module.

Proof. Assume first that $N$ is a finitely generated $R$-module and let $S$ be a zero-dimensional $T$-overring of $R$. Then $N \cap S$ is an Artinian $R$-module, hence an Artinan $S$-module. Thus, to show that $S$ is Artinian, it suffices to show that $S /(N \cap S)$ is an Artinian $S$-module, or equivalently, that $S /(N \cap S)$ is an Artinian ring. Now $N \cap S$ is the nilradical of $S$, so $S /(N \cap S)$ is a zerodimensional reduced subring of $T / N$, a ring with only finitely many idempotents since the map $e \rightarrow e+N$ is known to be a bijection of the idempotents of $T$ onto the idempotents of $T / N$ (cf. [L, p. 72]). Consequently, $S /(N \cap S)$ 
has only finitely many idempotents, and part (3) of Proposition 2.9 implies that $S /(N \cap S)$ is Artinian.

Conversely, assume that $(R, T)$ satisfies (ZA). Result 3.1 shows that $T$ has only finitely many idempotents. The ring $S=R+N$ is a zero-dimensional $T$-overring of $R$, and hence is Artinian. Therefore $N^{k}=(0)$ for some $k \in \mathbb{Z}^{+}$, and to show that $N$ is a finitely generated $R$-module, it suffices to show that $N^{i-1} / N^{i}$ is a finitely generated $R$-module for $2 \leq i \leq k$. Now $N^{i-1} / N^{i}$ is finitely generated as an $S$-module and as an $S / N$-module, where $S / N=$ $(R+N) / N \simeq R /(N \cap R)$. Since $(N \cap R) N^{i-1} \subseteq N^{i}$, it follows that $N^{i-1} / N^{i}$ is a finitely generated $R$-module, as we wished to show.

Since a module $M$ over an Artinian ring is finitely generated if and only if $M$ has finite length, we note that in the statement of (3.2), the condition that $N$ is finitely generated as an $R$-module can be replaced by the condition that $N$ has finite length as an $R$-module.

In the statement of Proposition 3.3, use is made of the following result proved in [ $\left.\mathrm{GH}_{4}\right]$. If $\left\{S_{\lambda}\right\}_{\lambda \in \Lambda}$ is a nonempty family of zero-dimensional subrings of a ring $S$, then $\bigcap_{\lambda} S_{\lambda}$ is zero-dimensional. This implies that if $R$ is a subring of $S$ such that $R$ admits a zero-dimensional $S$-overring, then there exists a unique minimal zero-dimensional $S$-overring $R^{0}$ of $R$.

Proposition 3.3. Suppose that $R$ is a subring of $S$ and that $R$ admits a zerodimensional $S$-overring. Let $R^{0}$ denote the minimal zero-dimensional $S$-overring. Then the following are equivalent.

(1) $(R, S)$ satisfies $(\mathrm{ZA})$.

(2) $S$ has only finitely many idempotents, $R^{0}$ is Artinian, and $N(S)$ is a finitely generated $R^{0}$-module (or equivalently, $N(S)$ is an $R^{0}$-module of finite length).

Proof. Since it is clear that $(R, S)$ and $\left(R^{0}, S\right)$ simultaneously satisfy (ZA), Proposition 3.3 follows immediately from Result 3.1 and Theorem 3.2.

Corollary 3.4. Assume that $\operatorname{char} R \neq 0$. Then $R$ satisfies (ZA) if and only if $R$ has only finitely many idempotents and $N(R)$ is finite.

Proof. Let $\pi$ denote the prime subring of $R$. By assumption $\pi$ is a finite ring. Thus Corollary 3.4 follows immediately from Theorem 3.3 and the fact that, by definition, $R$ satisfies (ZA) if and only if $(\pi, R)$ satisfies (ZA) .

In the statement of our next result, Theorem 3.5, we use the fact noted in Result 2.8 that an indecomposable ring of characteristic zero that contains a zero-dimensional subring contains $\mathbb{Q}$.

Theorem 3.5. Suppose $S$ is an indecomposable ring of characteristic zero such that $S$ admits a zero-dimensional subring. Then $S$ satisfies (ZA) if and only if $N(S)$ is a finite-dimensional vector space over $\mathbb{Q}$.

Proof. Suppose first that $N(S)$ is a finite-dimensional vector space over $\mathbb{Q}$, and let $R$ be a zero-dimensional subring of $S$. By Remark 2.5(i), $R$ is quasilocal. Proposition 2.3 shows that $\mathbb{Q}$ is a subring of $R$. To show that $R$ is Artinian, it suffices, by Cohen's Theorem, to show that $N(R)$ is finitely generated as an ideal of $R$ (cf. Theorems 1 and 2 of $\left[\mathrm{C}_{2}\right]$ ). This follows since $N(R)$ is a $\mathbb{Q}$ subspace of $N(S)$, hence finitely generated as a vector space over $\mathbb{Q}$, and hence finitely generated as an ideal of $R$. 
Suppose, conversely, that $S$ satisfies (ZA). Then $\mathbb{Q}+N$, where $N=N(S)$, is a zero-dimensional subring of $S$, and hence is Artinian. Choose $k \in \mathbb{Z}^{+}$so that $N^{k}=(0)$. For $1 \leq i \leq k-1, N^{i} / N^{i+1}$ is a finite-dimensional vector space over $(\mathbb{Q}+N) / N \simeq \mathbb{Q}$, so $N$ is also finite dimensional as a vector space over $\mathbb{Q}$.

Theorem 3.6. A ring $S$ satisfies (ZA) if and only if $S$ is a finite direct product of indecomposable rings, each of which satisfies (ZA).

Proof. If $S$ satisfies (ZA), then $S$ has only finitely many idempotents by Result 3.1 , and hence is a direct sum

$$
S=S_{1} \oplus \cdots \oplus S_{k}
$$

where the $S_{i}$ are indecomposable as rings. By Remark 2.7, each $S_{i}$ has a zero-dimensional subring. Fix $i$ and a zero-dimensional subring $R_{i}$ of $S_{i}$; we wish to show that $R_{i}$ is Artinian. Thus, for $j \neq i$, choose a zero-dimensional subring $R_{j}$ of $S_{j}$. Then $R_{1} \oplus \cdots \oplus R_{k}$ is a zero-dimensional subring of $S$, hence Artinian, so in particular, $R_{i}$ is Artinian.

Conversely, assume that $S=S_{1} \oplus \cdots \oplus S_{k}$, where each of the rings $S_{i}$ satisfies (ZA). Again (2.7) implies that $S$ contains a zero-dimensional subring. Let $e_{i}$ denote the identity element of $S_{i}$, and let $R$ be a zero-dimensional subring of $S$. Then $R e_{i}$ is a zero-dimensional subring of $S_{i}$, and hence is Artinian, for each $i$. Thus $R e_{i} \oplus \cdots \oplus R e_{k}=R\left[e_{1}, \ldots, e_{k}\right]$ is Noetherian and a finitely generated $R$-module. Consequently $R$ is zero-dimensional and Noetherian by Eakin's Theorem [E], and hence is Artinian (cf., for example, [ZS, p. 203]).

We remark that Theorem 3.6 is a definitive result insofar as Corollary 3.4 and Theorem 3.5 provide equivalent conditions for an indecomposable ring to satisfy (ZA). The proof of Theorem 3.6 easily generalizes to yield the following relative version, which we label as Theorem 3.7 (cf. Theorem 3.2).

Theorem 3.7. Suppose $R$ is an Artinian subring of the ring $T$ and assume that $T$ is a finite direct sum of indecomposable ideals $T e_{1}, \ldots, T e_{n}$, where each $e_{i}$ is idempotent. Then $(R, T)$ satisfies (ZA) if and only if $\left(R_{i}, T e_{i}\right)$ satisfies (ZA) for each $i$.

The (ZA)-condition amounts to requiring that each zero-dimensional subring should be Noetherian. The next result, Proposition 3.8, gives necessary and sufficient conditions for each Noetherian subring to be zero dimensional or, equivalently, to be Artinian.

Proposition 3.8. Let $S$ be a ring with prime subring $\pi$. Each Noetherian subring of $S$ is Artinian if and only if $\pi$ is finite and $S$ is integral over $\pi$, hence if and only if $S$ is hereditarily zero-dimensional.

Proof. If $\pi$ is finite and $S$ is integral over $\pi$, then each Noetherian subring of $S$ is zero-dimensional, hence Artinian.

Conversely, if each Noetherian subring of $S$ is Artinian, then $\pi$ is Artinian, hence finite. We show that $S$ is hereditarily zero-dimensional. Suppose not, and let $R$ be a subring of $S$ of positive dimension. Let $P_{0}<P_{1}$ be prime ideals of $R$, and choose $x \in P_{1}-P_{0}$. Then $\pi[x]$ is a Noetherian subring of $S$ of positive dimension since $x \in\left(P_{1} \cap \pi[x]\right)-\left(P_{0} \cap \pi[x]\right)$. This contradicts the fact 
that $\pi[x]$ is zero-dimensional. Therefore $S$ is hereditarily zero-dimensional, and $\left[\mathrm{GH}_{3}\right.$, Corollary 4.2] shows that $S$ is integral over $\pi$.

The question that Proposition 3.8 addresses can also be considered in a relative context. In this regard, a proof similar to that of (3.8) yields the following result.

Proposition 3.9. Suppose $R$ is a subring of a Noetherian subring of a ring $T$. Then each Noetherian T-overring of $R$ is Artinian if and only if $T$ is zerodimensional and $T$ is integral over each Noetherian $T$-overring of $R$; if $R$ itself is Noetherian, this amounts to saying that $R$ is Artinian and $T$ is integral over $R$.

\section{Closure of the Set of Artinian SUbrings UNDER INTERSECTION}

If $R$ is a reduced ring, then it is known that the set of Artinian subrings of $R$ is closed under arbitrary intersection. For a general ring $R$, it is known that an arbitrary intersection of zero-dimensional subrings is again zero dimensional $\left[\mathrm{GH}_{4}\right]$. Hence if $R$ satisfies $(\mathrm{ZA})$, then an arbitrary intersection of Artinian subrings of $R$ is again Artinian. In this section we show in Example 4.2 that the set $\mathscr{A}$ of Artinian subrings of $R$ need not, in general, be closed under finite intersection, and in Theorem 4.6 we show that $\mathscr{A}$ may be closed under finite intersection, but not under arbitrary intersection.

Proposition 4.1. Suppose $T$ is an Artinian local ring of the form $T=K+M$, where $K$ is a field, $M$ is the maximal ideal of $T$, and $M \neq(0)$.

(1) If $F$ is a subfield of $K$ and $V$ is an $F$-submodule of $M$ such that $V^{2} \subseteq V$, then $F+V$ is a zero-dimensional subring of $T$.

(2) Let $F$ and $V$ be as in the statement of (1). Then $F+V$ is Artinian if and only if $V$ is a finitely generated vector space over $F$.

(3) If $\left\{F_{\alpha}\right\}$ is a family of subfields of $K$ and if, for each $\alpha, V_{\alpha}$ is an $F_{\alpha^{-}}$ submodule of $M$ such that $V_{\alpha}^{2} \subseteq V_{\alpha}$, then $\bigcap_{\alpha}\left(F_{\alpha}+V_{\alpha}\right)=\left(\bigcap F_{\alpha}\right)+$ $\left(\bigcap V_{\alpha}\right)$.

Proof. Statement (1) is clear, and (3) follows because the sum $K+M$ is direct as a sum of abelian groups. We prove (2). Thus suppose $F+V$ is Artinian. Since $V$ is nilpotent, a proof similar to that given in the second part of the proof of Theorem 3.2 shows that $V$ is a finitely generated $F$-module. Conversely, if $V$ is a finitely generated $F$-module, then $F+V$ is a finite-dimensional, hence Artinian, vector space over $F$, so $F+V$ is an Artinian ring.

Example 4.2. Suppose $T=F(X)[Y] /\left(Y^{2}\right)=F(X)+F(X) y$, where $F$ is a field of characteristic zero and $y=Y+\left(Y^{2}\right)$. It is well known (cf., for example, [ $\mathrm{J}, \mathrm{p}$. 31, Exercise 5]) that $F\left(X^{2}\right) \cap F\left(X^{2}+X\right)=F$ in this case. Hence Proposition 4.1 implies that $R_{1}=F\left(X^{2}\right)+F(X) y$ and $R_{2}=F\left(X^{2}+X\right)+F(X) y$ are Artinian subrings of $T$ such that $R_{1} \cap R_{2}=F+F(X) y$ is not Artinian.

It is natural to ask about the converse of part (1) of Proposition 4.1-that is, to ask for conditions on $T$ in order that each zero-dimensional subring of $T$ should be of the form $F+V$. We answer this and a related question in Proposition 4.3. For ease of reference, we say that a subring of $T$ of the form $F+V$, as in part (1) of (4.1), is of splitting type. 
Proposition 4.3. Let $T$ be an Artinian local ring of the form $T=K+M$, where $K$ is a field and $M \neq(0)$ is the maximal ideal of $T$, and let $E$ be a subfield of $K$.

(a) The following conditions are equivalent.

(1) Each $T$-overring of $E$ is of splitting type.

(2) Each zero-dimensional T-overring of $E$ is of splitting type.

(3) $K / E$ is separable algebraic.

(b) Each zero-dimensional subring of $T$ is of splitting type if and only if $K$ is an absolutely algebraic field. If char $E=p \neq 0$ and $K$ is absolutely algebraic, then each subring of $T$ is of splitting type.

Proof. For the sake of reference in the proof, we note that if $f(X) \in E[X]$ and if $y=a+n$, where $a \in K$ and $n \in M$, then

$$
f(y)=f(a+n)=f(a)+f^{\prime}(a) n+h n^{2},
$$

where $h \in E[a, n] \subseteq T$.

We first prove (a). Clearly (1) implies (2). We show that (2) fails if (3) fails. Thus, if $K$ is not algebraic over $E$, we choose $a \in K$ transcendental over $E$ and $n \in M-(0)$. If $y=a+n$, then it follows from $(*)$ that $y$ is transcendental over $E$ and that each nonzero element of $E[y]$ is a unit of $T$. Therefore $E(y)$ is a zero-dimensional $T$-overring of $E$. Since $E(y)$ does not contain the nilpotent element $n$, it does not contain $y-n=a$, and hence $E(y)$ is not of splitting type. Next we assume that $K / E$ is algebraic but not separable. Let $\operatorname{char} E=p \neq 0$; replacing $E$ by an intermediate field if necessary, we may assume without loss of generality that $K / E$ is purely inseparable and $K \neq E$. We choose $a \in K-E$ of degree $p^{v}$ over $E$ and we choose $n \in M-(0)$ so that $n^{p^{v}}=0$. If $y=a+n$, then $y^{p^{v}}=0$, and it follows from $(*)$ that no polynomial over $E$ of degree less than $p^{v}$ has $y$ as a root. Consequently, $X^{p^{v}}-y^{p^{v}}$ is the minimal polynomial for $y$ over $E, E[y] \simeq E[a]$, and again $E[a]$ is a zero-dimensional $T$-overring of $E$. The argument given in the case where $K / E$ is transcendental again applies to show that $E[y]$ is not of splitting type. This completes the proof that (2) implies (3).

Now assume that condition (3) is satisfied and let $R$ be a $T$-overring of $E$. Since $T / E$ is integral, $R$ is zero-dimensional and quasilocal with maximal ideal $M \cap R$, and $M \cap R$ is nilpotent since $M$ is nilpotent. Hence, in the terminology of [N, p. 13], $R$ is a complete local ring which may not be Noetherian. Moreover, since $R /(M \cap R) \subseteq K$ is separable over $E$ it follows from Theorem 31.9 of [N] that there exists a subfield $F$ of $R$ such that $R=F+(M \cap R)$. If $y \in F$ and if we write $y=a+n$, where $a \in K$ and $n \in M$, then $E[y]$ is a field. Hence, the kernel of the natural map $E[X] \rightarrow E[y]$ is generated by an irreducible polynomial $f(X) \in E[X]$. Now $(*)$ implies that $f(a)=0$ since $f(a) \in F \cap M=(0)$, and hence $f(X)$ is the minimal polynomial for $a$ over $E$. However, since $K / E$ is separable, this implies that $f^{\prime}(a)$ is a unit of $T$. The equality $n=-f^{\prime}(a)^{-1} h n^{2}$ in $T$ implies that $n=0$. Therefore $y=a \in K$ and $F \subseteq K$. Therefore $R$ is of splitting type. This completes the proof of (a).

Statement (b) follows immediately from (a) and from the following observation. If $K / \mathbb{Q}$ is algebraic, then by part (i) of Remark 2.5 , each zero-dimensional subring $R$ of $T$ is quasilocal. The residue field of $R$ is a subfield of $K$, and hence has characteristic 0 , so Proposition 2.3 shows that $R$ contains $\mathbb{Q}$. 
Theorem 4.4. Let $T$ be an Artinian local ring of the form $T=K+M$, where $K$ is a field, $M$ is the maximal ideal of $T$, and $M \neq(0)$. Assume that $E$ is a subfield of $K$ such that $K / E$ is separable algebraic. (Thus each $T$-overring of $E$ is of splitting type.)

(1) The set of Artinian T-overrings of $E$ is closed under finite intersection if and only if for any subfields $E_{1}, E_{2}$ of $K$ containing $E$ and for any finite extensions $F_{1}, F_{2}$ of $E_{1}$ and $E_{2}$ in $K, F_{1} \cap F_{2}$ is a finite extension of $E_{1} \cap E_{2}$.

(2) The set of Artinian $T$-overrings of $E$ is closed under arbitrary intersection if and only if for each family $\left\{E_{\alpha}\right\}_{\alpha \in A}$ of subfields of $K$ containing $E$ and for each family $\left\{F_{\alpha}\right\}_{\alpha \in A}$, where $F_{\alpha}$ is a finite extension of $E_{\alpha}$ in $K, \bigcap_{\alpha} F_{\alpha}$ is a finite extension of $\bigcap_{\alpha} E_{\alpha}$.

Proof. We prove (1); the proof of (2) is similar. Thus, suppose first that $\left[\left(F_{1} \cap F_{2}\right):\left(E_{1} \cap E_{2}\right)\right]$ is finite if $F_{i} / E_{i}$ is a finite extension for $i=1,2$. If $R_{1}$ and $R_{2}$ are Artinian $T$-overrings of $E$, then the hypothesis and part (2) of Proposition 4.1 imply that $R_{i}=E_{i}+V_{i}$, where $E_{i}$ is a subfield of $K$ containing $E$ and $V_{i}$ is a finitely generated $E_{i}$-submodule of $M$ such that $V_{i}^{2} \subseteq V_{i}$. Moreover, $R_{1} \cap R_{2}=\left(E_{1} \cap E_{2}\right)+\left(V_{1} \cap V_{2}\right)$, and we wish to show that the $\left(E_{1} \cap E_{2}\right)$-module $V_{1} \cap V_{2}$ is finitely generated. Thus, let $\left\{m_{i}\right\}_{i=1}^{s}$ be a $K$-vector space basis for $M$. Since $V_{i}$ is a finite $E_{i}$-module, there exists a finite extension field $F_{i}$ of $E_{i}$ in $K$ such that $V_{i} \subseteq F_{i} m_{1}+F_{i} m_{2}+\cdots+F_{i} m_{s}$, so $V_{1} \cap V_{2} \subseteq\left(F_{1} \cap F_{2}\right) m_{1}+\cdots+\left(F_{1} \cap F_{2}\right) m_{s}$, a finitely generated vector space over $E_{1} \cap E_{2}$. Hence $V_{1} \cap V_{2}$ is also finitely generated, so that $R_{1} \cap R_{2}$ is again Artinian, as we wished to show.

To prove the converse, suppose $F_{1} \cap F_{2}$ is not finite dimensional over $E_{1} \cap E_{2}$ for some finite extensions $F_{i} / E_{i}, i=1,2$. Choose $m \in M-(0)$ such that $m^{2}=0$. Then $E_{1}+F_{1} m$ and $E_{2}+F_{2} m$ are Artinian $T$-overrings of $E$, but their intersection, $\left(E_{1} \cap E_{2}\right)+\left(F_{1} \cap F_{2}\right) m$, is not Artinian since $\left[\left(F_{1} \cap F_{2}\right):\left(E_{1} \cap E_{2}\right)\right]$ is not finite. This completes the proof of Theorem 4.4.

In our next result we use the concept of the Steinitz number of a field algebraic over a finite field [J, Exercise 6, p. 147]. Let $\left\{p_{i}\right\}_{i=1}^{\infty}$ be a labeling of the prime integers. If $K$ is a field algebraic over the prime field with $p$ elements, then the Steinitz number associated with the field $K$ is the infinite tuple $\left(a_{1}, a_{2}, \ldots, a_{n}, \ldots\right)$, where each $a_{i}$ is either a nonnegative integer or the symbol $\infty$, and $a_{i}$ is determined as follows. If $K$ contains a field with $p^{p_{i}^{n}}$ elements, but does not contain a field with $p_{i}^{p_{i}^{n+1}}$ elements, then $a_{i}=n$. If $K$ contains a field with $p^{p_{i}^{n}}$ elements for each nonnegative integer $n$, then $a_{i}=\infty$. Up to isomorphism, an absolutely algebraic field of characteristic $p$ is uniquely determined by its Steinitz number. Moreover, given any infinite tuple $\left(a_{1}, a_{2}, \ldots, a_{n}, \ldots\right)$, there exists an absolutely algebraic field of characteristic $p$ having this tuple as its Steinitz number. If $E$ and $F$ are two absolutely algebraic fields of nonzero characteristic that are both subfields of some field, then $E \subseteq F$ if and only if $b_{i} \leq c_{i}$, for all $i \in \mathbb{Z}^{+}$, where $\left(b_{1}, b_{2}, \ldots\right)$ is the Steinitz number of $E$ and $\left(c_{1}, c_{2}, \ldots\right)$ is the Steinitz number of $F$. Moreover, if $E \subseteq F$, then $F$ is finite over $E$ if and only if $b_{i}=c_{i}$ for all but finitely many positive integers $i$, and if $b_{i}<c_{i}$, then $c_{i}<\infty$-that is, if $b_{i}$ is finite, then $c_{i}$ is also finite. It also readily follows that if $E_{1}$ and $E_{2}$ are absolutely algebraic fields of nonzero characteristic that are both subfields of some field and if $E_{i}$ has Steinitz number $\left(b_{1 i}, b_{2 i}, \ldots\right)$, then the Steinitz number of 
$E_{1} \cap E_{2}$ is $\left(d_{1}, d_{2}, \ldots\right)$, where $d_{i}=\min \left\{b_{1 i}, b_{2 i}\right\}$. From these observations it follows that an absolutely algebraic field $K$ of positive characteristic satisfies the condition in part (1) of Theorem 4.4. We record this fact as Proposition 4.5.

Proposition 4.5. Suppose that $K$ is an absolutely algebraic field of positive characteristic. If $E_{1}$ and $E_{2}$ are subfields of $K$ and if $F_{i}$ is a finite extension of $E_{i}$ in $K$ for $i=1,2$, then $F_{1} \cap F_{2}$ is a finite extension of $E_{1} \cap E_{2}$.

Let $A$ be the field of algebraic numbers. In contrast to the situation for the algebraic closure of a finite field, we note that the subfields of $A$ do not satisfy the conclusion of Proposition 4.5. For example, let $b$ be the real cube root of 2 and let $\zeta$ be a complex primitive cube root of unity. The fields $\mathbb{Q}(b)$ and $\mathbb{Q}(\zeta b)$ are formally real, and hence are contained in real closed subfields $E_{1}$ and $E_{2}$ of $A\left[\mathrm{~J}\right.$, Theorem 3, p. 274]. We have $A=E_{1}[i]=E_{2}[i]$, so $\left[A: E_{1}\right]=\left[A: E_{2}\right]=2$. Now $E_{1} \neq E_{2}$ because $E_{1}\left[E_{2}\right]$ contains $\zeta$, and hence $E_{1}\left[E_{2}\right]$ is not formally real since $1+\zeta^{2}+\zeta^{4}=0$. It follows that $\left[A: E_{1} \cap E_{2}\right]>2$, and hence $\left[A: E_{1} \cap E_{2}\right]=\infty[\mathrm{J}$, Theorem 17, p. 316]. In this connection, a significant factor that distinguishes $A$ from the algebraic closure $L$ of a finite field is that each subfield of $L$ is Galois over the prime subfield, but $A$ fails to satisfy this property. Using basic facts of Galois theory, it can be shown in general that if $E$ is a subfield of a field $F$ and if each intermediate field is Galois over $E$, then the set of subfields of $F$ containing $E$ satisfies the conclusion of (4.5). Thus, this holds, for example, if $F$ is the abelian closure of $\mathbb{Q}$.

Using (4.4) and (4.5), we obtain the following result concerning closure of the set of Artinian subrings under intersection.

Theorem 4.6. Suppose $T$ is an Artinian local ring of the form $T=K+M$, where $K$ is an absolutely algebraic field of positive characteristic, $M$ is the maximal ideal of $T$, and $M \neq(0)$. Let $\left(a_{1}, a_{2}, \ldots\right)$ denote the Steinitz number of the field $K$. Then the set $\mathscr{A}$ of Artinian subrings of $T$ is closed under finite intersection. Moreover, $\mathscr{A}$ is closed under arbitrary intersection if and only if only finitely many of the entries $a_{i}$ are not zero. Thus, there exist infinite fields $K$ for which $\mathscr{A}$ is closed under arbitrary intersection, but the Steinitz number of such a field $K$ has only finitely many nonzero entries.

Proof. Proposition 4.1 and part (b) of Proposition 4.3 show that $\mathscr{A}$ consists of all rings $F+V$, where $F$ is a subfield of $K$ and $V$ is a finitely generated $F$ submodule of $M$ such that $V^{2} \subseteq V$. Proposition 4.5 and part (1) of Theorem 4.4 then imply that $\mathscr{A}$ is closed under finite intersection.

Assume that infinitely many of the entries $a_{i}$ in the Steinitz number of $K$ are not 0 . Let $F$ denote the subfield of $K$ that has Steinitz number $\left(b_{1}, b_{2}, \ldots\right)$ where $b_{i}$ is defined to be 0 if $a_{i}=0$, and to be 1 if $a_{i} \neq 0$. Let $E_{i}$ be the subfield of $F$ that has the same Steinitz number as $F$ except possibly in the $i$ th coordinate. If $b_{i}=1$, define the $i$ th coordinate of $E_{i}$ to be 0 , while if $b_{i}=0$, define $E_{i}$ to have the same Steinitz number as $F$, so that $E_{i}=F$. It is clear that each $E_{i}$ is a subfield of $F$ over which $F$ is finite algebraic. Moreover, $\bigcap_{i=1}^{\infty} E_{i}=\pi$, the prime subfield of $K$, and $F / \pi$ is not a finite extension. Thus, part (2) of Theorem 4.4 shows that $\mathscr{A}$ is not closed under arbitrary intersection if the Steintiz number of $K$ has infinitely many nonzero entries. 
For the converse we also use part (2) of Theorem 4.4. Assume that the Steinitz number of $K$ is such that $a_{i}=0$ for all but finitely many $i$. If $E \subseteq F$ are subfields of $K$ such that $F$ is finite over $E$, then as noted above, if the $i$ th coordinate of the Steinitz number of $E$ is finite, then the $i$ th coordinate of the Steinitz number of $F$ is also finite. It is therefore immediate from properties of the Steinitz number that the condition in part (2) of Theorem 4.4 is satisfied. Therefore $\mathscr{A}$ is closed under arbitrary intersection.

\section{RINGS WHOSE SET OF ARTINIAN SUBRINGS FORMS A DIRECTED FAMILY}

In $\left[\mathrm{GH}_{3}\right]$ it is shown that if a ring is the directed union of a family of zerodimensional subrings with finite spectra, then it is at least a set-theoretic union of Artinian subrings, and it is left there as an open question whether such rings are actually a directed union of Artinian subrings. Of course, if the set of Artinian subrings forms a directed family, then this follows automatically. We consider here the problem of determining conditions under which the set of Artinian subrings forms a directed family. We begin with an example to illustrate the fact that the set of Artinian subrings of a ring need not be directed.

Example 5.1. Let $F$ be a field and let $\left\{X_{i}\right\}_{i=1}^{\infty} \cup\left\{Y_{i}\right\}_{i=1}^{\infty}$ be a set of elements that are algebraically independent over $F$. Define

$$
T=F\left(X_{1}, X_{2}, \ldots\right)\left[Y_{1}, Y_{2}, \ldots\right] /\left(Y_{1}, Y_{2}, \ldots\right)^{2},
$$

and let $y_{i}$ denote the image of $Y_{i}$ in $T$. Let $R_{1}$ denote the subfield $F\left(X_{1}, X_{2}\right.$, $\ldots$ ) of $T$, and let $R_{2}=F\left(X_{1}+y_{1}, X_{2}+y_{2}, \ldots\right)$. Then $R_{2}$ is also a subfield of $T$ and $T=R_{1}\left[R_{2}\right]$, the compositum of the subrings $R_{1}$ and $R_{2}$. Since $R_{1}$ and $R_{2}$ are Artinian while $T$ is not Artinian, it follows that $T$ is a zero-dimensional quasilocal ring for which the Artinian subrings do not form a directed family.

Remark 5.2. In connection with Example 5.1, it is interesting to note that since the ring $T$ is zero-dimensional and quasilocal, it is expressible as a directed union of a family of Artinian subrings [ $\mathrm{GH}_{3}$, Corollary 5.5]. The distinction here is that in Example 5.1, the family of all Artinian subrings of $T$ is not directed. The lack of uniqueness of a coefficient field (cf. Remark 5.3) for the ring $T$ is a reason the Artinian subrings of $T$ fail to form a directed family. One may also construct a similar example of the form $T=K+M$, where $K$ is a field of transcendence degree one over its prime subfield and $M^{2}=(0)$.

Remark 5.3. Suppose $(T, N)$ is a quasilocal ring that contains a field. A coefficient field for $T$ is a subfield $K$ of $T$ such that $T=K+N$, or equivalently, such that $K$ maps surjectively onto the residue field $T / N$ under the canonical homomorphism of $T$ onto $T / N$. It is clear that if $K$ is a coefficient field for $T$, then $K$ is a maximal subfield of $T$. If $T$ is a complete local ring having the same characteristic as its residue field, it is well known that $T$ has a coefficient field $\left[C_{1}\right.$, Theorem 9]. In particular, if $T$ is an Artinian local ring that contains a field, then $T$ contains a coefficient field. Cohen also proves the following results (1)-(3) about coefficient fields of complete local rings [loc. cit., Theorem 10 and corollary].

(1) If $(R, M)$ is a complete local ring having residue field of characteristic zero, then any maximal subfield of $R$ is a coefficient field, and $R$ has a unique coefficient field if and only if $R / M$ is algebraic over $\mathbb{Q}$. 
(2) If char $R=p>0$ and $R / M$ is perfect, then $R$ has a unique coefficient field, and this coefficient field is $\bigcap_{n=1}^{\infty} R^{p^{n}}$, where $R^{p^{n}}=\left\{r^{p^{n}}: r \in R\right\}$.

(3) If $(S, N)$ is a complete local ring containing a field, if $(R, M)$ is a complete local subring of $S$ such that $N \cap R=M$, and if the field $R / M$ is perfect and $E$ is a coefficient field for $R$, then there exists a coefficient field $F$ for $S$ such that $E \subseteq F$.

(4) With notation and hypothesis as in (3), if $S / N$ is algebraic over $R / M$, then the coefficient field $F$ of $S$ containing $E$ is unique. If char $S=p>0$, then this is clear since in this case $S$ admits a unique coefficient field. If char $S=0$, the statement follows by the following argument. Let $L$ be any coefficient field of $S$ containing $E$. It follows as in the proof that (3) implies (1) in Theorem 4.3 that $L \subseteq F$, and hence $L=F$, as asserted.

From statements (3) and (4) of Remark 5.3, we obtain the following.

Result 5.4. Suppose $(T, N)$ is a zero-dimensional quasilocal ring containing a perfect field $E$ such that $T$ is integral over $E$, or equivalently, such that the field $T / N$ is algebraic over the canonical image of $E$. Then $T$ has a unique coefficient field containing $E$.

Proof. Let $\left\{R_{\alpha}\right\}$ be the family of finite integral extensions of $E$ in $T$. Then $\left\{R_{\alpha}\right\}$ is a directed family of Artinian (complete) local rings. The residue field of $R_{\alpha}$ is an algebraic extension of $E$ and hence is perfect. It follows from parts (3) and (4) of Remark 5.3 that there exists a unique coefficient field $F_{\alpha}$ of $R_{\alpha}$ containing $E$. Moreover, the set $\left\{F_{\alpha}\right\}$ is a directed family. Since $T=\bigcup_{\alpha} R_{\alpha}$, it also follows that $K=\bigcup_{\alpha} F_{\alpha}$ is a coefficient field of $T$ and is the unique coefficient field of $T$ containing $E$.

Remark 5.5. If $T, N$, and $E$ are as in Result 5.4 and if $R$ is any $T$-overring of $E$, then from (5.4) it follows that $R$ has a unique expression of the form $R=K+M$, where $K$ is a field containing $E$ and $M$ is the maximal ideal of $R$ (cf. Proposition 4.3).

Theorem 5.6. Suppose $(T, N)$ is a zero-dimensional quasilocal ring containing a perfect field $E$ such that $T$ is integral over $E$. Then the Artinian $T$-overrings of $E$ are closed under finite compositum. In particular, the Artinian T-overrings of $E$ form a directed family.

Proof. It suffices to show that if $R_{1}$ and $R_{2}$ are Artinian $T$-overrings of $E$, then $R_{1}\left[R_{2}\right]=R$ is Artinian. By Result 5.4, $T=K+N$, where $K$ is a field extension of $E$, and $R_{i}=F_{i}+M_{i}$, where $F_{i}$ is a field such that $E \subseteq$ $F_{i} \subseteq K$ and $M_{i}$ is the maximal ideal of $R_{i}, i=1,2$. Since $R_{i}$ is Artinian, $M_{i}$ is finite-dimensional as a vector space over $F_{i}$. Let $F=F_{1}\left[F_{2}\right]$ be the compositum of the subfields $F_{1}$ and $F_{2}$ of $K$. Since $K / E$ is algebraic, $F$ is a field. Moreover, if $\left\{x_{0}=1, \ldots, x_{r}\right\}$ is a vector space basis for $R_{1}$ over $F_{1}$, and $\left\{y_{0}=1, \ldots, y_{s}\right\}$ is a vector space basis for $R_{2}$ over $F_{2}$, then $\left\{x_{i} y_{j}\right\}_{i=0 j=0}^{r}$ is a set of generators for $R$ as an $F$-module. Therefore $R$ is finite dimensional as a vector space over $F$ and hence is Artinian.

Corollary 5.7. Suppose $(T, N)$ is zero-dimensional quasilocal ring such that either (1) $T / N$ is an algebraic extension of $\mathbb{Q}$, or (2) char $T=p$, a prime integer, and $T / N$ is algebraic over a finite field. Then the set of Artinian subrings of $T$ is closed under finite compositum. 
Proof. In case (1), Proposition 2.3 shows that any Artinian subring of $T$ contains $\mathbb{Q}$. Therefore Theorem 5.6, with $\mathbb{Q}=E$, applies. In case (2), Theorem 5.6 applies with $\pi=E$, where $\pi$ is the prime field with $p$ elements.

Remark 5.8. If $(T, N)$ is a quasilocal ring of characteristic $p^{n}$, where $p$ is a prime integer and $n>1$, then the prime subring $\pi$ of $T$ is isomorphic to $\mathbb{Z} / p^{n} \mathbb{Z}$, and $T$ admits no subring that is a field or even an integral domain, so there is no possibility of a coefficient field for $T$. In this case a coefficient ring for $T$ is defined to be a zero-dimensional local subring $T^{\prime}$ of $T$ such that $p T^{\prime}$ is the maximal ideal of $T^{\prime}$ and $T^{\prime} / p T^{\prime}=T / N$ under the canonical inclusion map of $T^{\prime} / p T^{\prime}$ into $T / N$. This definition of a coefficient ring is a slight modification of the way the term is used by Cohen in $\left[C_{1}, p .84\right]$, and agrees with the usage in [N, p. 106]. It is well known that if $(R, M)$ is an Artinian subring of $T$, then $R$ has a coefficient ring $R^{\prime}\left[C_{1}\right.$, Theorem $11, \mathrm{~N}$, Theorem 31.1]. Cohen also proves the following results about coefficient rings $\left[\mathrm{C}_{1}\right.$, Theorem 13].

(1) If $R$ is a complete local ring of characteristic $p^{n}$ and if the residue field of $R$ is perfect, then $R$ has a unique coefficient ring $R^{\prime}$.

(2) With $R$ as in (1), if $S$ is a complete local ring containing $R$ as a subring and dominating $R$, then any coefficient ring $S^{\prime}$ of $S$ contains $R^{\prime}$.

Result 5.9. Suppose $(R, M)$ is an Artinian local ring of characteristic $p^{n}$ and $R / M$ is a perfect field of characteristic $p$. Assume that $(T, N)$ is a quasilocal extension ring of $R$ such that $T$ is integral over $R$, or equivalently, such that $T / N$ is algebraic over $R / M$. Then $T$ has a coefficient ring $T^{\prime}$. Moreover, if $S$ is a T-overring of $R$, then $S$ has a unique coefficient ring $S^{\prime}$ and $S^{\prime}$ is a subring of $T^{\prime}$. In particular, $T^{\prime}$ is the unique coefficient ring of $T$.

Proof. Let $\left\{R_{\alpha}\right\}$ be the directed family of finite integral extensions of $R$ in $T$. Each $R_{\alpha}$ is Artinian with perfect residue field and by Remark 5.8, $R_{\alpha}$ has a unique coefficient ring $R_{\alpha}^{\prime}$. The set $\left\{R_{\alpha}^{\prime}\right\}$ is a directed family of finite extensions of the unique coefficient ring $R^{\prime}$ of $R$. Since $p R_{\alpha}^{\prime}$ is the unique maximal ideal of $R_{\alpha}^{\prime}$ for each $\alpha$, it follows that $T^{\prime}=\bigcup_{\alpha} R_{\alpha}^{\prime}$ has maximal ideal $p T^{\prime}$ and is an Artinian local PIR. Since $T=\bigcup_{\alpha} R_{\alpha}$ and $R_{\alpha}^{\prime} / p R_{\alpha}^{\prime}=R_{\alpha} / p R_{\alpha}$ for each $\alpha$, it follows that $T^{\prime} / p T^{\prime}=T / N$. Therefore $T^{\prime}$ is a coefficient ring for $T$. If $S$ is a $T$-overring of $R$, then the residue field of $S$ is an algebraic extension of $R / M$ and hence is perfect. If $\left\{R_{\beta}\right\}$ is the directed family of finite extensions of $R$ in $S$, then as observed above, each $R_{\beta}$ has a unique coefficient ring $R_{\beta}^{\prime}$ and $S^{\prime}=\bigcup_{\beta} R_{\beta}^{\prime}$ is a coefficient ring of $S$. By construction, it is clear that $S^{\prime}$ is a subring of $T^{\prime}$. To see that $S^{\prime}$ is the unique coefficient ring of $S$, let $S^{*}$ be a coefficient ring of $S$. Note that $S^{*} / p S^{*} \subseteq S^{\prime} / p S^{\prime}$ by part (4) of Remark 5.3. It follows that $S^{*} \subseteq S^{\prime}+p S^{*} \subseteq S^{\prime}+p\left(S^{\prime}+p S^{*}\right) \subseteq S^{\prime}+p^{2} S^{*}$, and by an easy induction argument that $S^{*} \subseteq S^{\prime}+p^{n} S^{*}=S^{\prime}$. By a symmetric argument $S^{\prime} \subseteq S^{*}$, so $S^{\prime}=S^{*}$. Therefore $S^{\prime}$ is the unique coefficient ring of $S$.

Theorem 5.10. Suppose $(T, N)$ is a zero-dimensional quasilocal ring of characteristic $p^{n}$ and $(R, M)$ is an Artinian local subring of $T$ such that $T$ is integral over $R$. Assume that $R / M$ is a perfect field of characteristic $p$, and denote by 
$R^{\prime}$ the unique coefficient ring of $R$. Then the Artinian T-overrings of $R^{\prime}$ are closed under finite compositum.

Proof. It suffices to show that if $S_{1}$ and $S_{2}$ are Artinian $T$-overrings of $R^{\prime}$, then the compositum $S=S_{1}\left[S_{2}\right]$ is Artinian. Since $R$ is integral over $R^{\prime}$ and $T$ is integral over $R, T$ is integral over $S$. Therefore $S$ is quasilocal. By Result 5.9, $S$ has a unique coefficient ring $S^{\prime}$. Moreover, if $S_{i}^{\prime}$ is the unique coefficient ring of $S_{i}$, then $S_{i}^{\prime} \subseteq S^{\prime}$ by Result 5.9. Therefore $S_{1}^{\prime}\left[S_{2}^{\prime}\right] \subseteq S^{\prime}$. Moreover, the field $S^{\prime} / p S^{\prime}$ is the compositum of its subfields $S_{1}^{\prime} / p S_{1}^{\prime}$ and $S_{2}^{\prime} / p S_{2}^{\prime}$. It follows that $S^{\prime} \subseteq S_{1}\left[S_{2}\right]+p S^{\prime}$ and hence that $S^{\prime} \subseteq S_{1}\left[S_{2}\right]+p^{i} S^{\prime}$ for each positive integer $i$. Since $p^{n} S^{\prime}=(0)$, we conclude that $S^{\prime}=S_{1}^{\prime}\left[S_{2}^{\prime}\right]$. To show $S$ is Artinian, it suffices to show that $S$ is a finitely generated $S^{\prime}$-module, and for this it suffices to show $S^{\prime}\left[S_{1}\right]$ and $S^{\prime}\left[S_{2}\right]$ are finitely generated $S^{\prime}$ modules. By symmetry, it is enough to show that $S^{\prime}\left[S_{1}\right]$ is a finitely generated $S^{\prime}$-module. Let $M_{1}$ denote the maximal ideal of $S_{1}$ and let $\left\{x_{1}, \ldots, x_{n}\right\}$ be a finite set of generators for $M_{1}$ as an ideal of the Artinian ring $S_{1}$. We show that $S^{\prime}\left[S_{1}\right]=S^{\prime}\left[x_{1}, \ldots, x_{n}\right]$. Since $S_{1}^{\prime} \subseteq S^{\prime}$ and $S_{1}=S_{1}^{\prime}+M_{1}$, we have

$$
S_{1} \subseteq S^{\prime}+M_{1}=S^{\prime}+x_{1} S_{1}+\cdots+x_{n} S_{1} .
$$

Substituting for $S_{1}$ in the right-hand side of this expression and iterating, we see that

$$
S_{1} \subseteq S^{\prime}\left[x_{1}, \ldots, x_{n}\right]+M_{1}^{k},
$$

for each positive integer $k$. Since $M_{1}$ is nilpotent, it follows that $S^{\prime}\left[S_{1}\right]=$ $S^{\prime}\left[x_{1}, \ldots, x_{n}\right]$, a finitely generated $S^{\prime}$-module, as we wished to show. This completes the proof of Theorem 5.10 .

Since a hereditarily zero-dimensional ring has absolutely algebraic residue fields [ $\mathrm{GH}_{3}$, Corollary 4.3], we have the following corollary of (5.7) and (5.10).

Corollary 5.11. Suppose $T$ is a hereditarily zero-dimensional ring with Spec $T$ finite. Then the set $\mathscr{A}$ of Artinian subrings of $T$ is directed, and in fact, $\mathscr{A}$ is closed under finite compositum.

Proof. $T$ is a finite direct sum $T_{1} \oplus \cdots \oplus T_{n}$, where $T_{i}$ as a ring is hereditarily zero-dimensional and quasilocal. Let $R$ and $S$ be Artinian subrings of $T$ and let $E$ be the set of idempotent elements of $T$. If $R$ and $S$ contain $E$, then $R=R_{1} \oplus \cdots \oplus R_{n}$ and $S=S_{1} \oplus \cdots \oplus S_{n}$, where $R_{i}$ and $S_{i}$ are subrings of $T_{i}$ for $1 \leq i \leq n$. Corollary 5.7 and Theorem 5.10 show that $R_{i}\left[S_{i}\right]$ is Artinian for each $i$, so $R_{1}\left[S_{1}\right] \oplus \cdots \oplus R_{n}\left[S_{n}\right]=R[S]$ is Artinian. In the general case, since $E$ is finite, the argument just given shows that $(R[S])[E]$, the compositum of $R[E]$ and $S[E]$, is Artinian. But since $(R[S])[E]$ is a finite integral extension of $R[S]$, it follows from Eakin's Theorem [E] that $R[S]$ is also Artinian.

\section{REFERENCES}

$\left[\mathrm{C}_{1}\right]$ I. S. Cohen, On the structure and ideal theory of complete local rings, Trans. Amer. Math. Soc. 59 (1946), 54-106.

$\left[\mathrm{C}_{2}\right]-$ Commutative rings with restricted minimum condition, Duke Math. J. 17 (1950), 27-42.

[E] P. Eakin, The converse to a well-known theorem on Noetherian rings, Math. Ann. 177 (1968), 278-282. 
[G $\left.\mathrm{G}_{1}\right]$ R. Gilmer, Integral domains with Noetherian subrings, Comment. Math. Helv. 45 (1970), 129-134.

$\left[\mathrm{G}_{2}\right]$ - Domains with integrally closed subrings, Math Japon. 16 (1971), 9-11.

$\left[\mathrm{G}_{3}\right] \_$, Multiplicative ideal theory, Marcel-Dekker, New York, 1972.

[GH $\left.{ }_{1}\right]$ R. Gilmer and W. Heinzer, Noetherian pairs and hereditarily Noetherain rings, Arch. Math. 41 (1983), 131-138.

$\left[\mathrm{GH}_{2}\right] \ldots$, On the imbedding of a direct product into a zero-dimensional commutative ring, Proc. Amer. Math. Soc. 106 (1989), 631-637.

$\left[\mathrm{GH}_{3}\right] \ldots$, Products of commutative rings and zero-dimensionality, Trans. Amer. Math. Soc. 331 (1992), 663-680.

$\left[\mathrm{GH}_{4}\right] \_$, Zero-dimensionality in commutative rings, Proc. Amer. Math. Soc. 115 (1992), 881893.

[H] J. Huckaba, Commutative rings with zero divisors, Marcel-Dekker, New York, 1988.

[J] N. Jacobson, Lectures in abstract algebra, vol. 3, Van Nostrand, Princeton, N.J., 1964.

[L] J. Lambek, Lectures on rings and modules, Blaisdell, Waltham, Mass., 1966.

[M] P. Maroscia, Sur les anneaux de dimension zero, Atti Accad. Naz. Lincei Rend. Cl. Sci. Fis. Mat. Natur. 56 (1974), 451-459.

[N] M. Nagata, Local rings, Interscience, New York, 1962.

[ $\left.\mathrm{W}_{1}\right]$ A. Wadsworth, Pairs of domains where all intermediate domains are Noetherian, Trans. Amer. Math. Soc. 195 (1974), 201-211.

[W $\left.\mathrm{W}_{2}\right] \ldots$, Hilbert subalgebras of finitely generated algebras, J. Algebra 43 (1976), 298-304.

[ZS] O. Zariski and P. Samuel, Commutative algebra, vol. I, Springer, New York, 1975. 32306

Department of Mathematics B154, Florida State University, Tallahassee, Florida

E-mail address: gilmer@math.fsu.edu

Department of Mathematics, Purdue University, West Lafayette, Indiana 47907

E-mail address: heinzer@math.purdue.edu 